\title{
Miniaturized 3D Printed Particulate Matter Sensor for Personal Monitoring
}

\author{
Yunqi Wang, Fidel Valega Mackenzie, Bastiaan Ingenhut, Arjen Boersma \\ TNO, Materials Solutions, High Tech Campus 25, 5656 AE, Eindhoven, the Netherlands \\ yunqi.wang@tno.nl
}

\begin{abstract}
:
The development of low-cost, small, lightweight portable particulate matter sensors with increased accuracy that can be worn permanently in work and health related environments is recently in high demand. A novel miniaturized sensing system which possesses the functions of separating and measuring the mass concentration of the aerosol particles is presented. The system comprises a plurality of microchannels to separate incoming particles depending on their size and integrates capacitance sensors in the channels to detect particulate matter. Simulations have been performed to optimize the design of the channel structures. Subsequently digital light projection 3D printing technique has been employed for manufacturing. The final design has been successfully used to demonstrate real-time detection of particles $(10 \mu \mathrm{m})$ in air. This approach enables the realization of next-generation personal portable devices for detecting dust, pollution, contaminants, among others, and can be utilized for early warning or real-time intervention applications.
\end{abstract}

Key words: particulate matter sensor, virtual impactor, airborne particles, low-cost, portable.

\section{Introduction}

Particulate matter sensors are widely used for measuring and/or detecting various classes of airborne particles in a fluid. Typically, larger devices are employed to accurately indicate the different fractions of particles in air, which is done conventionally by light scattering [1]. On the contrary, relatively small devices that utilize low-cost sensors provide a fairly poor accuracy and may for instance not be able to detect the particles or distinguish the particle size accurately. This work aims at the next step in the development of low-cost miniaturized wearable particulate matter sensors with increased accuracy, low energy consumption, limited nuisance for the users, and easily interpretable feedback.

The developed system comprises a plurality of microchannels that function as virtual impactor (VI) through which air is guided by means of a pump or fan. Inside the microchannels the incoming aerosol particles are sorted into different channels according to its size and mass. The detection of the particles in the channels in the $\mathrm{VI}$ is by means of integrated capacitance sensors.

\section{Design of Virtual Impactor}

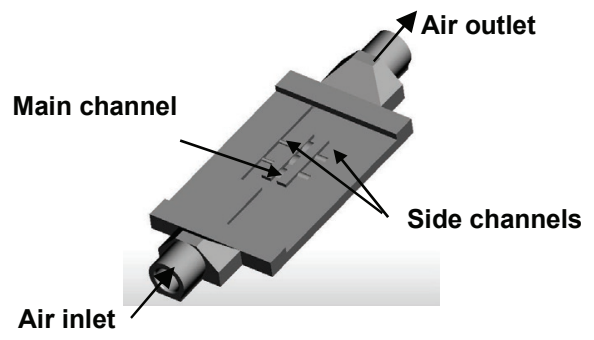

Fig. 1. Design of the VI. The large particles follow preferentially to the main channel. Smaller particles also deviate to the side channels.

Simulations were carried out to optimize the air flow conditions and the collection efficiency for particle separation in the fluidic channels. This involved parameter exploration under specific boundary conditions in addition to key geometrical features. The designed prototype (see Fig. 1) was manufactured by using the socalled digital light projection 3D printing technique which is capable of fabricating relatively complex structures while facilitating rapid and numerous modifications.

The validation of the particle separation in the sensor was proved by employing a series of 
fluorescent particles $(2,5,10 \mu \mathrm{m})$ in a gas flow through the printed $\mathrm{VI}$. Moreover ridges were arranged along the channels' width to guide particles towards the detectors. For comparison purposes the ratio of the number of particles in the side channels to the total number through the inlet was used as measure for the efficiency. As such this ratio reflects the degree of particle separation which upon testing agrees qualitatively with the results obtained through simulations (see Fig. 2). The fraction of the particles present in the side channels at higher flow rate is lower than that at lower rate. In general, the greater particles are mainly carried along the main channel due to their larger inertial force, while smaller particles might follow towards the lateral channels in more or less degree depending on the applied flow.

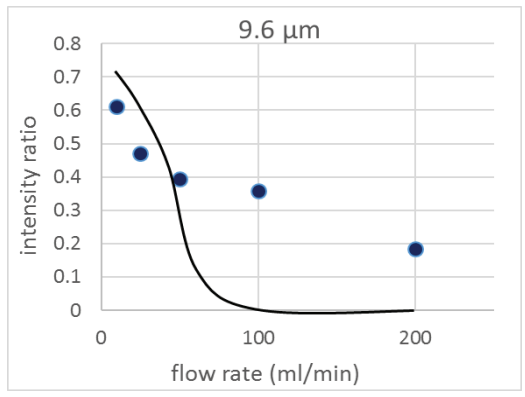

Fig. 2. Effect of flow rate on particle separation efficiency. Simulation (line) and experimental (dot) data for $9.6 \mu \mathrm{m}$ melamine particles.

\section{Demonstration of Sensing System}

Static tests were preliminarily performed to experimentally prove the principle of capacitive detection of single microparticles. Particles of $10 \mu \mathrm{m}$ diameter were deposited on three interdigitated electrodes to be simultaneously measured (see Fig. 3). In the dynamic systemlevel test, a particle detector chip was integrated to the 3D printed VI (see Fig. 4). The particle detector was designed based on capacitive electrodes with a sensitivity of $10 \mathrm{aF}$. The detector chip $20 \times 12 \mathrm{~mm}$ containing 12 integrated electrodes. VIs were designed to have ridges positioned according to prior flow studies and taking into account the electrode positions on the detector chip. A pump was connected to the outlet of the impactor and the particles were led through a thin pipe to the inlet. Dynamic tests were performed using silica particles $(<10 \mu \mathrm{m})$. In the case when a stream of single particles passes over the electrodes, each particle generates a positive pulse of capacitance change $\Delta \mathrm{C}$, as shown in Fig. 5 . At $\mathrm{t}$ $=4 \mathrm{~min}$ the sensor was exposed to a greater concentration of silica particles resulting in an instantaneous and enhanced peaked response.
The response signal returned to the baseline once there was no particle exposure, which demonstrates the reversibility of the sensor.

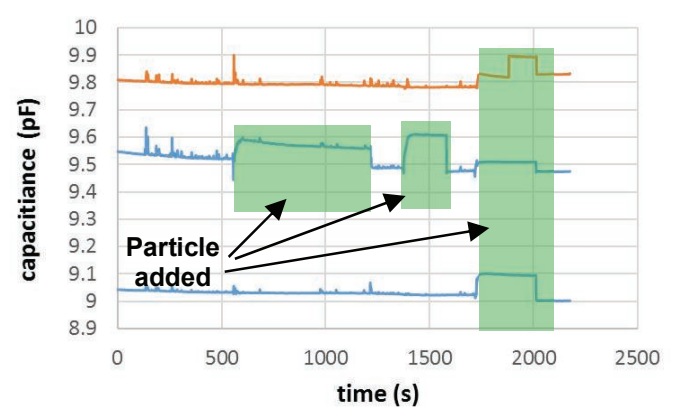

Fig. 3. Static test by measuring the deposition of particles on three electrodes simultaneously.

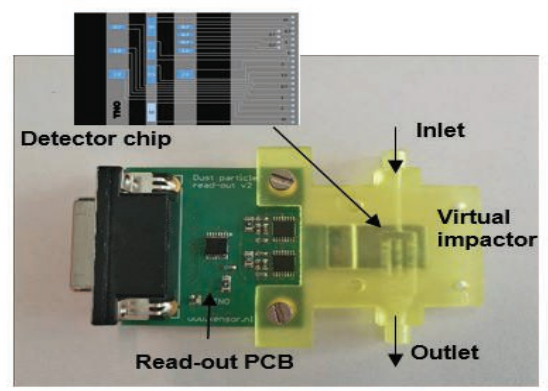

Fig. 4. Integrated particle detectors and VI.

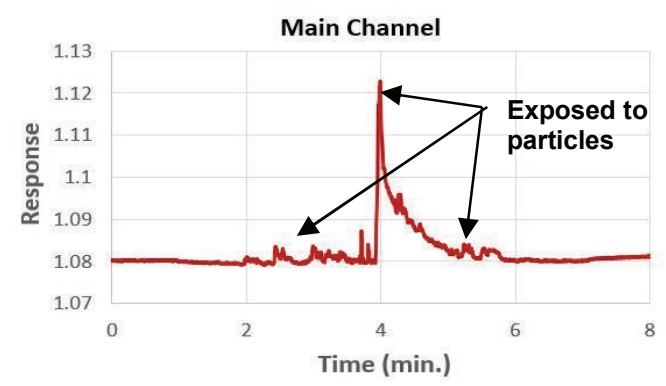

Fig. 5. Particles are detected in dynamic test.

\section{Conclusions}

A miniature aerosol sensing system based on a method for detecting particles in a fluid by means of separation was designed, fabricated and tested. The demonstrated system can be potentially applied to personal health monitoring at work place. It provides the possibility of combining a flexible way to separate particles by size while measuring parameters such as mass, volume or dielectric constant of the particles. All included in a portable particle sensor capable of also warning on the harmful health effects of exposure to particulate matter.

\section{References}

[1] M. Stephens, N. Turner, J. Sandberg, Particle Identification by Laser-induced Incandescence in a Solid-state Laser Cavity, Applied Optics 42, 3726-3736 (2003); doi: 10.1364/AO.42.003726 